Bull. Austral. Math. Soc.

VoL. 42 (1990) [141-144]

\title{
EQUIPARTITION OF CONVEX BODIES
}

\author{
PAUL R. ScOTT
}

We show that a compact convex body in $E^{n}$ cannot be partitioned by $n+1$ hyperplanes into $2^{n+1}-1$ subsets of equal measure, thus generalising a result in the plane due to R.C. and E.F. Buck.

\section{INTRODUCTION}

Let $K$ be a compact, convex body in Euclidean $n$-space, $E^{n}$, having measure $m K$. We consider the problem of partitioning $K$ using $n+1$ hyperplanes. If the hyperplanes have no point in common, they determine a simplex $\Delta$, and in this case $K$ will be partitioned into $2^{n+1}-1$ (possibly empty) subsets. In [1], R.C. and E.F. Buck prove:

Lemma 1. In $E^{2}$, no convex set $K$ can be partitioned by three lines into seven subsets of equal area.

We shall prove the analogue in $n$ dimensions.

THEOREM 1. For $n \geqslant 2$, no convex body $K$ in $E^{n}$ can be partitioned by $n+1$ hyperplanes into $2^{n+1}-1$ subsets of equal measure.

Apart from the simplex $\Delta$ itself, we observe that each of the subsets of $K$ corresponds in a natural way to a proper face of $\Delta$ - the face of largest dimension which it has in common with $\Delta$. With this understanding we shall refer to facet sets, edge sets and vertex sets.

We shall in fact prove the following stronger version of Theorem 1.

THEOREM 2. For $n \geqslant 2$, a convex body $K$ in $E^{n}$ is partitioned by $n+1$ hyperplanes into $2^{n+1}-1$ non-empty subsets. Then there exist four subsets comprising $\Delta$, a facet set, an edge set, and a vertex set which do not have equal measure.

\section{Proof of THEOREM 2}

The subsets described in Theorem 2 will shortly be specified more explicitly. We shall assume that these subsets have equal measure 1 , and arrive at a contradiction.

Received 28th September 1989

Copyright Clearance Centre, Inc. Serial-fee code: 0004-9729/90 \$A2.00+0.00. 
Let $h$ denote the hyperplane determining facet $f$ of $\Delta$, and let $v^{*}$ be the vertex of $\Delta$ not contained in $h$. We shall use the notation $C_{x}$ to denote the (finite) cone, $\operatorname{conv}\left(v^{*} \cup x\right)$, having apex $v^{*}$ and base $x$. Thus $\Delta=C_{f}$.

Let $F$ be the facet set associated with facet $f$. The $n$ rays through $v^{*}$ containing the edges of the simplex $\Delta$ meet the boundary of $K$ in points $t_{1}, \ldots, t_{n}$ say. By the convexity of $K$, the hyperplane $h^{*}$ determined by these points either supports or intercepts $F$. Hence there exists another hyperplane $h^{\prime}$ which (a) is parallel to $h^{*}$, (b) is separated from $\Delta$ by $h^{*}$ (perhaps not strictly), and (c) determines with $f$ and the determining hyperplanes of $\Delta$ passing through $v^{*}$, a new set of measure 1 (the same as $m F$ ).

Let $v$ be a vertex of $\Delta$ which is closest to $h^{\prime}$, and let $V$ denote the corresponding vertex set. Since $V$ and $F$ are separated by the $n-1$ hyperplanes containing the edge $v^{*} v$ of $\Delta$, the sets $F \cap h^{\prime}$ and $V \cap h^{\prime}$ have at most a single point in common - the point where the ray $\overrightarrow{v^{*} v}$ meets the boundary of $K$. Because $h^{\prime}$ supports or intercepts $F$, it follows from the convexity of $K$ that $h^{\prime}$ supports or bounds $V$.

Let now $E$ denote the edge set associated with $v^{*} v$, and define $e=E \cap h$. Since $E$ is convex, the cone $C_{e}$ is strictly contained in $E$, and $m C_{e}<1$. (The cone $C_{e}$ cannot coincide with $E$, else the vertex set $V^{*}$ would be empty.) We replace the set $e$ by another set $d$ in the hyperplane $h$, where $d$ is obtained by enlarging $e$ about the vertex $v$ using an enlargement of scale factor $\lambda>1$. The value of $\lambda$ is chosen so that $m C_{d}=1$, and clearly $d \supset e$. Let $f^{\prime}, e^{\prime}, d^{\prime}$ denote the projections of $f, e, d$ respectively from $v^{*}$ onto hyperplane $h^{\prime}$. From the convexity of $K$, the vertex set $V$ is a subset of $C_{e^{\prime}} ;$ since $C_{e^{\prime}} \subset C_{d^{\prime}}$, we deduce that $V \subset C_{d^{\prime}}$.

We now come to the final step in the construction. The vertex $v$ was chosen closest to $h^{\prime}$. We replace the hyperplane $h$ by a new hyperplane $h^{\prime \prime}$ which passes through $v$ and is parallel to $h^{\prime}$. Let $f^{\prime \prime}=h^{\prime \prime} \cap C_{f^{\prime}}, d^{\prime \prime}=h^{\prime \prime} \cap C_{d^{\prime}}$. If $h^{\prime \prime} \neq h$, the choice of $v$ ensures that the flat $h^{\prime \prime} \cap h$ has no point in common with the relative interior of the simplicial facet $f$. (For, if the flat $h^{\prime \prime} \cap h$ strictly separated two vertices of the facet $f$, one of those vertices would be closer to $h^{\prime}$ than $v$.) Hence $C_{f} \subset C_{f}^{\prime \prime}$, so $m C_{f} \leqslant m C_{f}^{\prime \prime}$ and $m\left(C_{f^{\prime}} \backslash C_{f^{\prime \prime}}\right) \leqslant m F=1$. Further, since $\Delta$ and $V$ are 'vertically opposite' sets with common point $v$, the hyperplane $h^{\prime \prime}$ which bounds or supports $\Delta$ also bounds or supports $V$. Similarly, $h^{\prime \prime}$ supports or intercepts $C_{d}$. Hence $m C_{d} \prime \leqslant m C_{d}=1$, and $m\left(C_{d^{\prime}} \backslash C_{d^{\prime \prime}}\right) \geqslant m\left(C_{d^{\prime}} \backslash C_{d}\right) \geqslant m V$.

Summing up, we now have cones $C_{f^{\prime}}$ and $C_{d^{\prime}}$ with common apex $v^{*}$ and bases lying in the same hyperplane $h^{\prime}$. These cones are partitioned by a hyperplane $h^{\prime \prime}$ parallel to the bases into cones $C_{f^{\prime \prime}}, C_{d^{\prime \prime}}$, and corresponding frustrums $B_{f}$ and $B_{d}$. Further, $m C_{f^{\prime \prime}} \geqslant 1, m C_{d^{\prime \prime}}<1, m B_{f} \leqslant 1$, and $m V \leqslant m B_{d}$. 
By properties of similarity,

$$
\frac{m C_{f^{\prime \prime}}}{m B_{f}}=\frac{m C_{d^{\prime \prime}}}{m B_{d}}<\frac{m C_{d^{\prime \prime}}}{m V}
$$

from which we deduce that $m V<1$.

\section{Some Extensions}

From the proof it is clear that the following variant of the theorem is also true.

COROLLARY. If the measures of $\Delta$, the vertex sets, the edge sets, and the facet sets are $d, v, e$, and $f$ respectively, then $d v<e f$.

Thus if $v=e=f=1$, then $d<1$. In the plane case, this led to the conjecture that $d \leqslant 1 / 8$, in fact attained for a triangle with partitioning lines parallel to the sides ([1]). This conjecture was established by Sholander [3], and later by Eggleston [2].

Also in the plane case, Buck and Buck established that if six of the sets determined by the three lines have equal area, then the seventh (unequal) set has to be $\Delta$. It is easy to establish the $n$-dimensional analogue.

LEMMA 2. If $n+1$ hyperplanes partition a convex body $K$ in $E^{n}$ into $2^{n+1}-1$ subsets of which $2^{\text {n+1 }}-2$ have equal measure, then the subset with measure different from the others is $\Delta$.

Proof: Let $\Delta$ have facets $f_{1}, \ldots, f_{n+1}$, and let $v_{j}$ be the vertex of $\Delta$ not lying in $f_{j}(1 \leqslant j \leqslant n+1)$. We consider the families of subsets of $K$ of the type which occur in the above proof, given by $\left\{\Delta, F_{i}, V_{j}, E_{i j}\right\}$, where $i=1,2, \ldots, n+1$ and for each value of $i, j$ takes some value $1 \leqslant j \leqslant n+1, j \neq i$. We assert that $\Delta$ is the only member all the families have in common. Clearly by choice the $F_{i}$ are all different. Since $j \neq i$, no $V_{j}$ can be common to all the families. And $E_{i j}\left(=E_{j i}\right)$ can be common to at most two families; hence for $n \geqslant 2, E_{i j}$ can not be common to all the families. Now if all subsets of $K$ but one have equal measure, and no four subsets in any of the above families all have equal measure, we deduce that $\Delta$ must be the subset with measure different from the others.

Let $n+1$ hyperplanes partition the $n$-dimensional convex set $K$ as above, and let $M(n, k)$ denote the maximal number of subsets of equal measure which can be determined in this way. Then we have shown that for all $n \geqslant 2, M(n, k) \leqslant 2^{n+1}-2$. Equality holds here for $n=2$, but experiment suggests that even for $n=3$ the bound can probably be improved.

\section{REFERENCES}

[1] R.C. Buck and E.F. Buck, 'Equipartition of convex sets', Math. Mag. 22 (1948), 195-198. 
[2] H.G. Eggleston, Problems in Euclidean space: application of convexity, pp. 126-129 (Pergamon Press, 1957).

[3] M. Sholander, 'Proof of a conjecture by R.C. and E.F. Buck', Math. Mag. 24 (1950), 8-10.

Department of Mathematics

University of Adelaide

Adelaide SA 5001

Australia 